\title{
Téoros
}

Revue de recherche en tourisme

\section{Les conditions d'une relation bénéfique entre tourisme et événementiel}

\author{
Le cas de Deauville, station balnéaire normande
}

\section{Nathalie Alexandre-Bourhis, Chantal Rouvrais-Charron et Marc Bourhis}

Volume 32, numéro 1, 2013

URI : https://id.erudit.org/iderudit/1036661ar

DOI : https://doi.org/10.7202/1036661ar

Aller au sommaire du numéro

Éditeur(s)

Université du Québec à Montréal

ISSN

0712-8657 (imprimé)

1923-2705 (numérique)

Découvrir la revue

Citer cet article

Alexandre-Bourhis, N., Rouvrais-Charron, C. \& Bourhis, M. (2013). Les conditions d'une relation bénéfique entre tourisme et événementiel : le cas de Deauville, station balnéaire normande. Téoros, 32(1), 123-132.

https://doi.org/10.7202/1036661ar
Résumé de l'article

De nombreuses collectivités territoriales font le choix, depuis quelques années, de développer une politique événementielle. L'objectif de cet article est de s’interroger sur la pertinence d'une telle stratégie de développement économique, c'est-à-dire sur les conditions d'une relation mutuellement bénéfique entre tourisme et événementiel, afin de renforcer l'attractivité d'un territoire. Fondée sur une étude de cas, l'analyse proposée se situe à l'échelle d'une ville, Deauville, station balnéaire normande. De type qualitatif, le dispositif méthodologique mis en oeuvre est basé sur des entretiens avec les principaux acteurs institutionnels du territoire étudié. Après une première partie consacrée à l'étude des travaux de recherche réalisés sur ce thème et une présentation du contexte dans lequel évoluent les territoires, les éléments nécessaires à la fédération des énergies et à la mise en place d'infrastructures sont mis en évidence. La réflexion menée permet, d'une part, d'identifier certains risques et points d'achoppement de ce type de stratégie et, d'autre part, de formuler des recommandations d'actions généralisables à d'autres cas de figure.
Ce document est protégé par la loi sur le droit d'auteur. L'utilisation des services d'Érudit (y compris la reproduction) est assujettie à sa politique d'utilisation que vous pouvez consulter en ligne.

https://apropos.erudit.org/fr/usagers/politique-dutilisation/ 


\section{Les conditions d'une relation bénéfique entre tourisme et événementiel

\author{
Le cas de Deauville, station balnéaire normande
}

\author{
Nathalie ALEXANDRE-BOURHIS \\ Maître de conférences en sciences de gestion \\ Département des techniques de commercialisation \\ Université de Caen Basse-Normandie (France) \\ nathalie.bourhis@unicaen.fr
}

\author{
Chantal ROUVRAIS-CHARRON \\ Maître de conférences en sciences de gestion \\ Département des techniques de commercialisation \\ Université de Caen Basse-Normandie (France) \\ chantal.rouvrais-charron@unicaen.fr
}

\author{
Marc BOURHIS \\ Maître de conférences associé \\ Université de Caen Basse-Normandie (France) \\ marc.bourhis@unicaen.fr
}

RÉSUMÉ: De nombreuses collectivités territoriales font le choix, depuis quelques années, de développer une politique événementielle. L'objectif de cet article est de s'interroger sur la pertinence d'une telle stratégie de développement économique, c'est-à-dire sur les conditions d'une relation mutuellement bénéfique entre tourisme et événementiel, afin de renforcer l'attractivité d'un territoire. Fondée sur une étude de cas, l'analyse proposée se situe à l'échelle d'une ville, Deauville, station balnéaire normande. De type qualitatif, le dispositif méthodologique mis en œuvre est basé sur des entretiens avec les principaux acteurs institutionnels du territoire étudié. Après une première partie consacrée à l'étude des travaux de recherche réalisés sur ce thème et une présentation du contexte dans lequel évoluent les territoires, les éléments nécessaires à la fédération des énergies et à la mise en place d'infrastructures sont mis en évidence. La réflexion menée permet, d'une part, d'identifier certains risques et points d'achoppement de ce type de stratégie et, d'autre part, de formuler des recommandations d'actions généralisables à d'autres cas de figure.

Mots-clés : Attractivité, territoire, destination, événement, développement, station balnéaire.

De nombreuses collectivités locales font le choix, depuis quelques années, de développer une politique événementielle. Ainsi voit-on fleurir des congrès, des salons et des événements d'envergure nationale ou internationale, qu'ils soient économiques, sportifs ou culturels. Ces manifestations semblent représenter, aux yeux des institutionnels, l'un des leviers économiques les plus opportuns des années 2010. En effet, les retombées de tels événements sont perceptibles sur le plan économique et social, ainsi que du point de vue de l'image.

À l'instar d'un produit ou d'un service, une destination touristique comme une station balnéaire traverse, au cours du temps, des étapes clés que l'on peut envisager sous l'angle du concept de «cycle de vie» (Butler, 1980) : elle naît, se développe, parvient au stade de la maturité, puis vieillit (Weiermair, 2006). Pour éviter d'entrer dans la phase de déclin, elle doit faire l'objet d'une politique lui permettant de se renouveler sans pour autant trahir son identité. L'organisation d'événements (festivals, fêtes, etc.) représente l'une des voies choisies par certaines municipalités pour apporter du dynamisme à leur ville.
La problématique de ce travail de recherche se focalise sur les questionnements suivants : quelles forces particulières les événements ont-ils pour attirer un public? En quoi les événements constituent-ils un facteur d'attractivité et de démarcation pour une collectivité territoriale? Il s'agit d'appréhender les conditions d'une relation mutuellement bénéfique entre tourisme et événementiel.

Cet article s'intéresse à la stratégie de développement d'un territoire de taille modeste, celui de Deauville, station balnéaire normande. Pour continuer à être présente dans l'esprit du grand public et des entreprises et demeurer à l'avant-scène, cette collectivité territoriale a fait le choix de se développer par la mise en œuvre de ressources dynamiques.

\section{Cadre théorique de l'étude}

Selon Augier (2009 : 4), la définition d'un grand événement, rejoignant celle proposée par Getz (2008), est « une manifestation qui, concernant principalement un secteur d'activité (sport, culture, économie), a des répercussions territoriales de tous ordres (création de valeur, effet d'image, facteur de 
cohésion sociale, divertissement) et, à un titre ou à un autre, un impact international (venue de touristes étrangers, effet d'image, réplication de l'événement à l'étranger) bénéfique pour le pays ou le territoire d'accueil». Deauville dispose de ressources spécifiques : une image internationale d'élégance, des équipements sportifs et culturels d'importance et un patrimoine naturel, culturel et architectural (555 villas classées), comme le mentionnent tous les descriptifs et ouvrages sur cette ville de Basse-Normandie (Gandin, 2009). Répondre à la question de recherche relative à l'alliance entre tourisme et événementiel, afin de développer l'attractivité d'un territoire, nous amène à nous référer à deux courants théoriques.

La définition d'Augier (2009) met l'accent sur les externalités positives engendrées par un événement. Dans le cas d'une collectivité territoriale, l'organisation d'événements constitue l'une des orientations possibles pour réussir à se démarquer durablement. On distingue trois principales catégories d'événements destinés au grand public : les événements sportifs, les événements culturels, artistiques ou historiques (conférences, expositions, festivals, etc.), de même que les événements commerciaux (foires, salons). Cette définition recouvre des réalités très différentes. Cela va de la manifestation locale au grand événement qui suppose un concept initial fort et fédérateur. Le tourisme est aujourd'hui moins centré sur les ressources naturelles (soleil, mer et plage, par exemple); on recherche davantage une consommation d'ordre symbolique ou sensoriel, comme la visite de lieux associés à des idées ou à des événements particuliers, ou le partage d'impressions visuelles, de sons et de goûts (Claveau, 2005; Hetzel, 2002; Holbrook et Hirschman, 1982; Marteaux et Mencarelli, 2005; OECD, 2009).

Le cadre conceptuel mobilisé dans cette recherche s'appuie ainsi, d'une part, sur la théorie des ressources dynamiques et, d'autre part, sur la pensée stratégique moderne. Par ses traits singuliers et ses atouts, le cas de Deauville s'inscrit dans l'approche par les «ressources». Cette théorie se base sur l'existence de ressources hétérogènes et idiosyncrasiques appartenant à une organisation, comme une ville, qui lui permettent de déjouer la concurrence (Penrose, 1963; Ghertman et Isnard, 1999). Ainsi, les organisations ont-elles la possibilité de capturer des rentes si elles disposent de ressources difficiles à imiter, non disponibles, non transférables et non substituables (Durand, 1998; Mechin, 2001 : 94). Le courant de recherche des ressources dynamiques est une hybridation des principes fondateurs de l'approche fondée sur les ressources (resource-based view) et de l'école évolutionniste.

L'approche fondée sur les ressources, inspirée des travaux de Penrose (1963), met l'accent sur les ressources de l'organisation, qu'elles soient tangibles ou intangibles, dans une perspective statique. Quant à l'école évolutionniste, elle prend en compte les changements économiques et technologiques de l'environnement dans lequel évoluent l'organisation et ses ressources propres (Mechin, 2001). Le courant des ressources dynamiques retenu accepte donc, d'une part, qu'une ressource possédée par une organisation évolue dans le temps et soit fonction d'un environnement mouvant. D'autre part, il met en lumière les constituants d'un avantage concurrentiel détenu par l'organisation qui, en outre, est dotée de compétences et de savoir-faire dans l'exercice de son activité. En effet, une collectivité territoriale, face à un environnement sociologique, économique et technologique évolutif, est amenée à renforcer et à renouveler ses ressources. Concomitamment, elle mobilise l'ensemble de ses compétences, notamment organisationnelles, pour amener toutes ses parties prenantes (élus, responsables de structures sportives ou culturelles, population, etc.) à contribuer au maintien de son avantage concurrentiel (Carroll et Buchholtz, 1989; Caroll, 1999; Mercier, 2001). La prise en compte de ces deux dimensions se réfère à l'étude des capacités d'une organisation à adapter son fonctionnement au dynamisme de son environnement. Nous postulons que, forte de son histoire et de ses ressources structurelles, naturelles et culturelles difficiles à imiter, la ville de Deauville peut développer une politique événementielle s'appuyant sur son savoir-faire organisationnel.

Par ailleurs, le cas de Deauville, alliant tourisme et événementiel, s'inscrit dans le courant de recherche de la pensée stratégique moderne (Marchesnay, 2002). Le développement de cette ville peut être considéré comme résultant de la combinaison de trois données : ses ressources dynamiques, son histoire et l'esprit d'entreprise, individuel ou collectif, qui anime les acteurs de ce territoire. Pour que Deauville dispose d'un avantage distinctif durable dans le temps, il convient de veiller à la symbiose de ces trois exigences. Dès lors, l'expansion de la ville, axée sur l'organisation d'événements favorisant le tourisme, reposerait également sur une construction volontariste de compétences acquises de façon incrémentale au fil du temps.

\section{Méthodologie de la recherche}

Le dispositif expérimental mis en œuvre est de nature qualitative. Ce choix méthodologique semble le plus adapté pour décrire, comprendre et traduire une politique de développement, dans un milieu spécifique tel qu'une ville. La collecte de données s'appuie sur des sources documentaires (documents internes, revue de presse) et des entretiens semi-directifs réalisés auprès de vingt acteurs principaux : cinq à la mairie, à la communauté de communes et au Centre international de Deauville (CID), trois dans les équipements équestres (Pôle international du cheval (PIC) et hippodromes) et deux à l'office de tourisme. Il s'agit d'élus (maires, maires adjoints ou élus communautaires) et de salariés (directeurs, directeurs de la communication, directeurs commerciaux ou chargés de communication). Il convient de préciser que des parties prenantes de la politique événementielle de la ville, comme les résidents, les touristes ou les commerçants, n'ont pas été sollicitées. Cet éclairage de la problématique nécessiterait une autre étude.

Aussi, en fonction de leur statut et du poste occupé, les personnes interrogées ont-elles répondu à quatre questions ouvertes portant sur la politique touristique de la ville de Deauville, le rôle joué dans cette politique, son impact sur le développement du territoire, ainsi que ses limites et difficultés. Dans une démarche qualitative, une importance est accordée aux entretiens avec les acteurs participant au développement de la collectivité territoriale (Alexandre-Bourhis et al., 2009). La parole de ces derniers étant considérée comme 
ILLUSTRATION 1 : Situation géographique de Deauville (Normandie, France) (source : adapté de istgeo.ac-aix-marseille.fr).

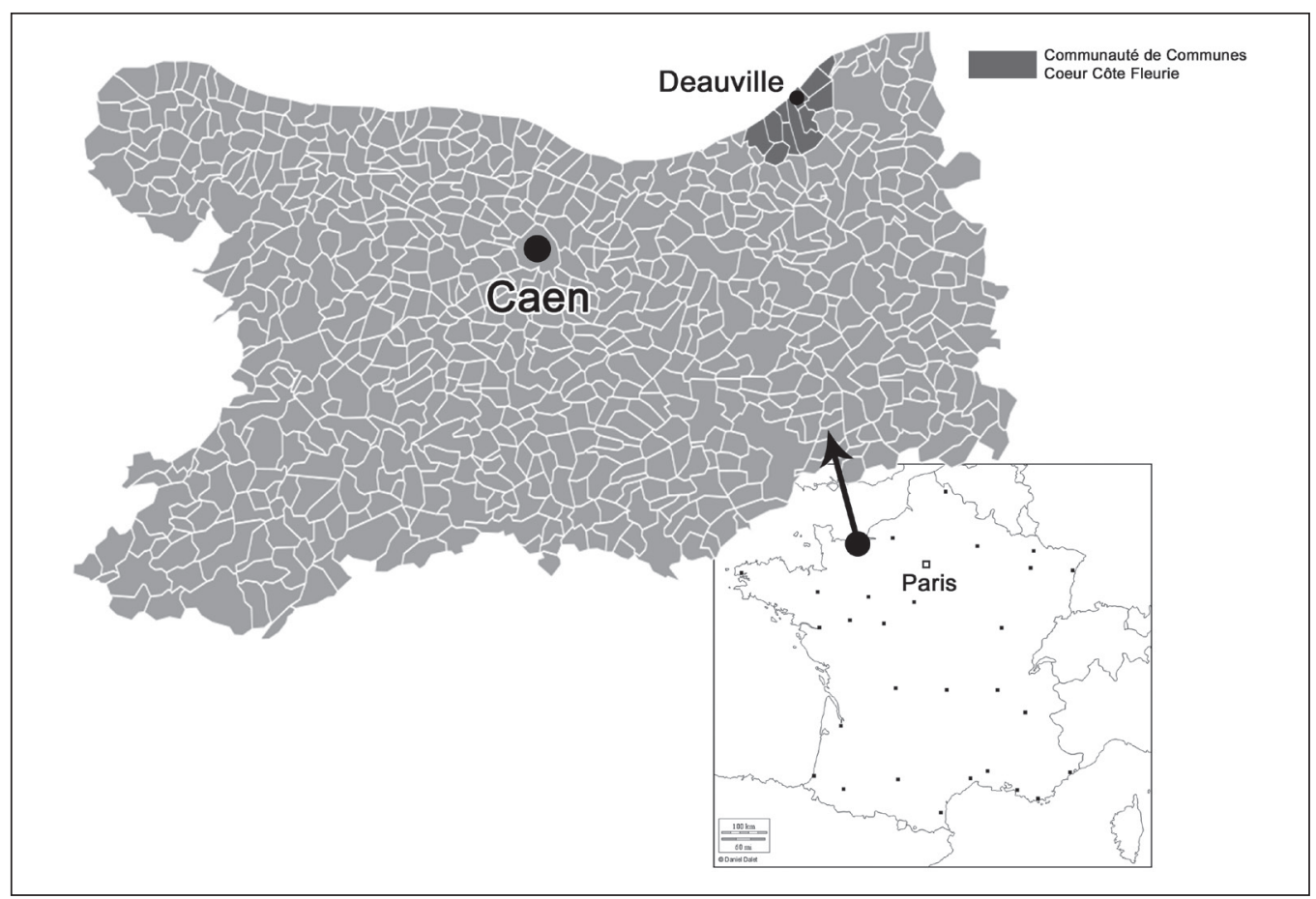

fondamentale, le cadre d'analyse est celui de l'acteur réseau (actor-network theory) (Akrich et al., 2006). En effet, cette méthodologie compréhensive, qualifiée de «sociologie de la traduction", postule que des actants disparates, aussi bien humains que techniques, s'unissent et tendent à faire converger leurs volontés dans un but commun; ils forment alors un acteur réseau. En outre, elle vise à construire une complémentarité entre les analyses scientifiques, mobilisant des modèles théoriques et des méthodologies, et les analyses d'acteurs s'adossant sur leur expérience professionnelle.

L'analyse des entretiens s'appuie sur le traitement textuel de leur contenu. Il s'agit de s'attacher au sens véhiculé par les discours, afin de mettre au jour des systèmes de représentation, des éléments de consensus et des divergences. Les données ont été codées selon les thèmes retenus, ces derniers étant un condensé de significations (Paillé et Mucchielli, 2003). Enfin, les résultats obtenus ont permis de dégager des enseignements pour une politique de développement touristique reposant sur l'événementiel.

\section{Étude du cas : Deauville, la destination d'hier à aujourd'hui}

Un regard vers l'histoire est incontournable si l'on souhaite appréhender la stratégie de développement d'un territoire sur le long terme. La ville de Deauville (3 962 habitants en 2010 selon l'INSEE (2013) et plus de 30000 en période estivale) se situe en Normandie, non loin des plages du Débarquement, sur la façade maritime du Pays d'Auge, à l'entrée de l'estuaire de la Seine (voir illustration 1), région dans laquelle les villégiatures d'été en bord de mer sont nées au milieu du $\mathrm{XIX}^{\mathrm{e}}$ siècle (Gandin, 2009). Comme c'est fréquemment le cas pour les villes balnéaires, l'histoire des stations de la Côte
Fleurie est le plus souvent associée à des personnes, comme le duc de Morny à Deauville. Dès la création d'une station de ce type, trois constructions sont toujours réalisées : un casino, un grand hôtel et un établissement de bains. Autour de ces édifices structurants prennent place les villas. Par ailleurs, l'existence d'une station balnéaire se doit de répondre aux attentes d'une société de plaisir, dont le séjour est une fête permanente. Depuis 153 ans, Deauville, surnommée par certains la «perle de la Normandie», jouit d'une réputation d'élégance, car elle a toujours été fréquentée par de nombreuses célébrités, issues du monde du cinéma, de la littérature ou de la haute couture. Certains, en l'associant à Trouville qui la jouxte, parlent de la «Riviera des Parisiens» en raison de sa proximité avec la capitale (deux heures de Paris par l'autoroute ou le train, et un aéroport pour des visiteurs plus lointains). Au-delà de son attrait d'élégance et de glamour, l'histoire de Deauville est liée à la filière équine (le duc de Morny ayant édifié un hippodrome, en 1864, avant même qu'une église soit construite) et au nautisme. En effet, de nombreuses manifestations hippiques internationales y sont organisées (courses, tournois de polo). Les ventes aux enchères de chevaux, qui s'y déroulent depuis 1887, attirent des acheteurs du monde entier. Quant au nautisme, Deauville est aussi, par sa situation sur la Manche, une étape incontournable pour les plaisanciers, qu'ils viennent de Grande-Bretagne, de la mer du Nord ou des îles anglo-normandes.

La présentation de Deauville impose de la situer dans la communauté de communes à laquelle elle appartient d'un point administratif et géographique. Celle-ci, nommée Cœur Côte Fleurie, regroupe onze petites villes et villages (Bénerville, Blonville, Deauville, Saint-Arnoult, Saint-Pierre-Azif, Touques, Tourgéville, Trouville, Vauville, Villers-sur-Mer et Villerville) 


\begin{tabular}{|c|c|}
\hline \multicolumn{2}{|c|}{$\begin{array}{c}\text { Tableau 1: Opinion des participants à l'étude quant à la politique } \\
\text { sur le territoire }\end{array}$} \\
\hline $\begin{array}{l}\text { Structures } \\
\text { concernées }\end{array}$ & $\begin{array}{l}\text { Question : Pouvez-vous parler de la politique } \\
\text { touristique de Deauville? }\end{array}$ \\
\hline Mairie & $\begin{array}{l}\text { Élus : } \\
\text { "Elle est basée sur les événements. Ils entraînent } \\
\text { des retombées économiques, créent de la cohésion } \\
\text { sociale et sont des outils stratégiques pour aménager } \\
\text { le territoire.» } \\
\text { «Depuis sa création, Deauville est mariée avec le } \\
\text { cheval.» } \\
\text { «La culture est une attente forte des touristes, donc } \\
\text { l'offre de Deauville est axée sur les événements } \\
\text { culturels, mais aussi, depuis toujours, sur le monde } \\
\text { équestre et les courses.» } \\
\text { "Le challenge, c'est d'être tous ensemble capables de } \\
\text { réaliser des actions concrètes qui correspondent aux } \\
\text { choix que nous avons faits.» } \\
\text { Salariés : } \\
\text { «Ici, les deux mots-clés sont sans doute cheval et } \\
\text { culture.» } \\
\text { "Notre objectif, c'est qu'il se passe toujours quelque } \\
\text { chose à Deauville.» } \\
\text { "Deauville, grâce à notre politique, c'est une } \\
\text { destination toute l'année et pas seulement l'été ou les } \\
\text { week-ends.» }\end{array}$ \\
\hline Office de tourisme & $\begin{array}{l}\text { "La politique touristique de la ville est entièrement } \\
\text { tournée vers l'événementiel.» } \\
\text { "Les axes forts sont le cheval et la culture.» } \\
\text { "Deauville est la vitrine internationale du cheval.» } \\
\text { "Deauville doit continuellement s'adapter à l'évolution } \\
\text { des attentes des touristes.» }\end{array}$ \\
\hline $\begin{array}{l}\text { Centre international } \\
\text { de Deauville (CID) }\end{array}$ & $\begin{array}{l}\text { «Elle est fondée sur l'événementiel et donc s'appuie } \\
\text { très fortement sur le CID.» } \\
\text { "Le CID, c'est un centre des congrès très important } \\
\text { pour une si petite ville ; c'est le principal outil de } \\
\text { Deauville pour accueillir le tourisme d'affaires et les } \\
\text { événements culturels majeurs, comme les festivals de } \\
\text { cinéma.» }\end{array}$ \\
\hline $\begin{array}{l}\text { Pôle international } \\
\text { du cheval (PIC) et } \\
\text { hippodromes }\end{array}$ & $\begin{array}{l}\text { «À Deauville, ce sont les événements qui sont mis en } \\
\text { avant; ; le PIC a été construit dans cette logique. On } \\
\text { est avant tout un lieu où des événements équestres } \\
\text { doivent être proposés à un public d'avertis, mais aussi } \\
\text { au grand public.» } \\
\text { "Deauville organise un nombre incalculable } \\
\text { d'événements toute l'année.» } \\
\text { "Dans les hippodromes, nous allongeons la saison } \\
\text { d'été en organisant des courses plus tard, en octobre.» }\end{array}$ \\
\hline $\begin{array}{l}\text { Communauté } \\
\text { de communes }\end{array}$ & $\begin{array}{l}\text { «Deauville est la ville centre de notre communauté } \\
\text { de communes. Elle joue un rôle moteur au niveau } \\
\text { événementiel tout au long de l'année. Cela permet } \\
\text { d'attirer les touristes et aussi d'inciter les résidents } \\
\text { secondaires à rester.» } \\
\text { «Notre objectif est aussi d'attirer les touristes vers } \\
\text { l'arrière-pays, pour découvrir le Pays d'Auge et ses } \\
\text { villages typiques.» }\end{array}$ \\
\hline
\end{tabular}

Source : entretiens des auteurs.

dans lesquels vivent 20794 habitants permanents (INSEE, 2013) et jusqu’à 120000 en période estivale. Deauville dispose de capacités d'accueil et d'équipements de loisirs : 2000 chambres dans un rayon de deux kilomètres, allant de la résidence parahôtelière aux grands hôtels de luxe, un palais des congrès de $18000 \mathrm{~m}^{2}$, un casino, deux hippodromes, un complexe équestre, un pôle omnisports, des parcours de golf et un centre de thalassothérapie. Accessible par l'autoroute, la station est également dotée d'un aéroport et de deux ports.

\section{Tableau 2: Exemples d'événements organisés à Deauville}

\begin{tabular}{l|l}
\hline Types d'événements & Exemples \\
\hline Grands événements & G27 en 2008, G3 en 2010, G8 en 2011 \\
& Women's forum (sorte de« Davos féminin ») \\
\hline Festivals et & Festival du cinéma américain (60 000 festivaliers en \\
événements culturels & $2013)$ \\
& Festival du film asiatique \\
& Festival de Pâques et Août musical (musique classique) \\
& Festival de photographie \\
& Salon livres \& musiques (10 000 visiteurs en 2012) \\
& 150 ans de Deauville en 2010 (365 événements \\
& culturels) \\
& Expositions, rencontres, ateliers et visites patrimoniales \\
& Saison culturelle de la ville \\
& Programmation culturelle du CID et du Groupe Lucien \\
& Barrière (théâtre, concerts, danse, spectacles) \\
\hline Événements & Courses de plat et d'obstacle de notoriété mondiale \\
équestres & (135 000 visiteurs en 2012), compétitions (66 dont \\
& quatre de niveau international en 2013), spectacles, \\
& stages de préparation olympique des équipes de \\
& France, vente de chevaux (4' rang mondial pour la \\
& vente de pur-sang), etc. \\
\hline Autres manifestations & $\begin{array}{l}\text { Tournois de basket-ball et de volley-ball de niveau } \\
\text { international, stages de préparation aux compétitions }\end{array}$ \\
sportives & internationales (Jeux olympiques de Londres et de Rio), \\
& triathlon international, régates (Laser Race, Cowes \\
Deauville), étape du Tour de France à la voile en 2013, \\
départ de la course du Figaro en 2014
\end{tabular}

\section{Résultats de l'analyse des entretiens}

L'exploitation des sources documentaires et des entretiens semi-directifs conduit à des résultats, dont la présentation s'articulera autour des trois thèmes abordés. Nous en déduirons les effets de synergie qui semblent intervenir dans le cas de Deauville. La transcription des entretiens réalisés a mené à l'élaboration de tableaux de synthèse regroupant des citations textuelles évocatrices des opinions énoncées (tableaux 1 et 3 à 5 ).

L'événement en qualité de levier de croissance pour une ville est le premier thème qui se dégage de l'analyse des entretiens. Pour le maire de Deauville, «les événements représentent un outil stratégique pour aménager les territoires, produire des retombées économiques et créer de la cohésion sociale» (voir tableau 1).

Les personnes interrogées s'accordent pour reconnaître que l'objectif principal est d'organiser ou d'accueillir des événements de toutes tailles. Un élément fédérateur de la politique événementielle est le cheval, qui est représenté sous toutes ses facettes (courses, concours, spectacles, ventes de pur-sang, élevage, entraînement de chevaux de course, compétitions d'attelage et de polo, etc.). Par ailleurs, même si des manifestations très variées sont organisées, la priorité est donnée à la culture (OTD, 2013). La mairie justifie ce choix en s'appuyant 
sur les études qui mettent en lumière les avantages d'un renforcement de la relation entre tourisme et culture (OECD, 2009), tels que :

- l'amélioration de l'image : par exemple, le Festival du cinéma américain, créé en 1975 à Deauville, valorise le cinéma indépendant et n’est plus uniquement dédié au lancement des productions américaines. Cette évolution a permis à l'événement d'être reconnu au niveau international par les producteurs, les réalisateurs et les acteurs;

-l'acceptabilité sociale (Caron-Malenfant et Conraud, 2009) : les événements doivent fédérer toute la population. À l'occasion des 150 ans de Deauville en 2010, les habitants ont apporté une large contribution par leur participation en tant que spectateurs, mais aussi par leur investissement dans l'organisation des manifestations;

- le soutien de l'activité culturelle et le surcroît d'innovation et de créativité. Un exemple récent cité est celui du concours photo de la $25^{\mathrm{e}}$ heure, qui «offre un espace-temps virtuel d'une heure, d'une nuit exceptionnelle, permettant d'exprimer ses talents de photographe. Une occasion unique et annuelle pour s'offrir une tranche de vie supplémentaire, pour créer, observer, arpenter Deauville...» (Ville de Deauville, 2013).

Le tableau 2 présente des exemples d'événements récurrents ou uniques organisés à Deauville. Il met notamment en évidence le nombre important de manifestations culturelles proposées au public.

Les retombées de la politique événementielle constituent le deuxième thème tiré de l'analyse des entretiens. Les acteurs du territoire de Deauville sollicités lors de cette étude déclarent que, bien que disposant de nombreux atouts distinctifs, la ville doit continuellement s'adapter à l'évolution des attentes des touristes. L'analyse des entretiens fait ressortir l'idée selon laquelle Deauville aurait su ne pas se reposer sur son histoire atypique mais, au contraire, aurait été capable de se remettre en cause. Selon le maire, la problématique serait de réussir à se départir de l'image de frivolité qui caractérise la cité normande, sans trahir sa quintessence (Augier, 2008). En effet, un audit réalisé en 2008 par le cabinet Comanaging, spécialisé en marketing territorial, a confirmé l'image de frivolité de la ville, parfois perçue comme vieillissante. On oppose souvent Deauville, la cité «bling bling», à sa voisine, Trouville, jugée plus familiale et authentique (Polony, 2010). Dans les propos recueillis, les élus interrogés ne contredisent pas cette image, mais cherchent à la faire évoluer. Selon l'un des responsables du CID : " Le G8 et le Women's Forum, par exemple, témoignent de notre réussite en la matière. Nous accueillons aussi des événements très sérieux». Le maire affirme : «Je souhaite faire en sorte que les héritages du passé perdurent, tout en développant sans cesse une politique événementielle. La culture et le tourisme d'affaires sont des leviers économiques essentiels.»

Cependant, pour qu'un cercle vertueux s'instaure véritablement et que l'association entre tourisme et événementiel soit réussie, il faut organiser des événements « dans le respect d'une image où culture et innovation s'allient avec les valeurs traditionnelles de la ville pour renouveler le public touristique et faire vivre la ville tout au long de l'année», selon un élu.

\begin{tabular}{l|l}
\multicolumn{2}{l}{ Tableau 3: Opinion des participants à l'étude quant à l'impact de la } \\
\\
politique sur le territoire
\end{tabular}

Source : entretiens des auteurs.

En ce qui concerne l'impact de la politique touristique, les responsables interrogés affirment tous qu'il est économique et d'image (voir tableau 3), ce que confirment les études citées comme celle de Novamétrie (2007) sur la notoriété et l'attractivité du Calvados (Calvados Tourisme, 2007). Selon cette étude, parmi les trois événements les plus connus du département, deux sont Deauvillais : le Festival du film américain et les événements équestres. Au plan économique, les alentours de la ville bénéficient de l'activité plus grande (résidences secondaires davantage occupées, activité des gîtes et chambres d'hôtes, etc.) (CCCCF, 2010). 


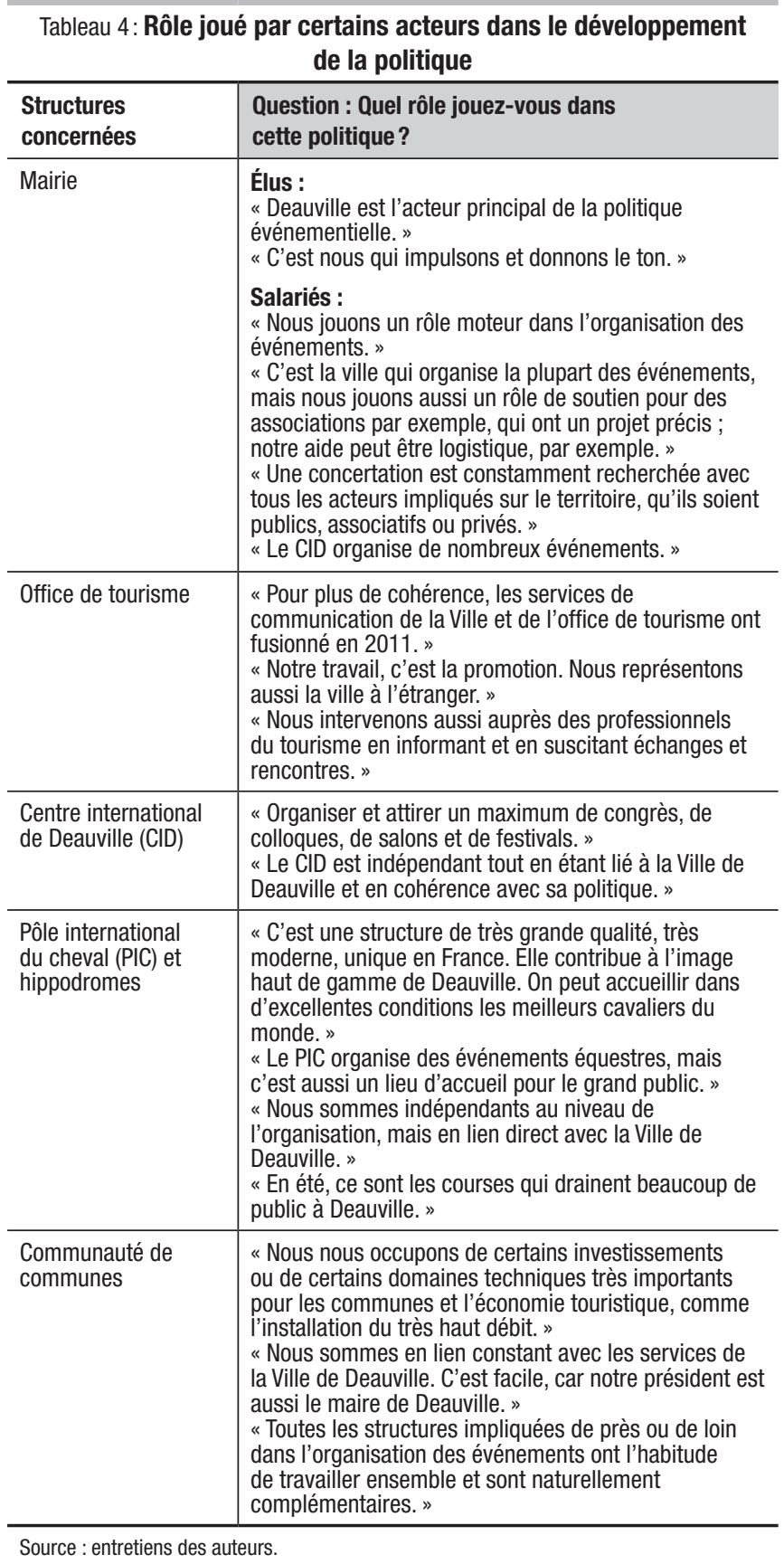

Source : entretiens des auteurs.

Enfin, les propos tenus précédemment doivent être tempérés par la présence de limites et de difficultés rencontrées lors de la mise en place d'une politique événementielle. Ceci est l'objet du troisième thème dégagé de l'analyse du discours des élus et des professionnels. En effet, il s'avère que l'application d'une politique événementielle semble pouvoir se heurter à trois types de difficultés. Tout d'abord, concernant l'organisation des événements, les décisions sont susceptibles d'être prises par tous les acteurs suivant les cas de figure, sans que cela semble poser de problème particulier

\begin{tabular}{|c|c|}
\hline \multicolumn{2}{|c|}{$\begin{array}{c}\text { Tableau 5: Opinion des participants à l'étude quant aux difficultés } \\
\text { de mise en place de la politique }\end{array}$} \\
\hline $\begin{array}{l}\text { Structures } \\
\text { concernées }\end{array}$ & $\begin{array}{l}\text { Question : Quelles sont, selon vous, les limites et } \\
\text { les difficultés de cette politique? }\end{array}$ \\
\hline Mairie & $\begin{array}{l}\text { Élus : } \\
\text { «II ne faut jamais s'arrêter de créer. L'événement, c'est } \\
\text { la marque de fabrique de la ville. » } \\
\text { "Les investissements s'imposent régulièrement. » } \\
\text { Salariés : } \\
\text { "C'est une politique très exigeante, car il faut toujours } \\
\text { innover, se remettre en cause, entretenir les réseaux. » } \\
\text { "La réussite dépend directement de l'entente qui } \\
\text { existe depuis toujours avec le Groupe Lucien Barrière, } \\
\text { c'est un partenariat historique. » } \\
\text { "Il faut communiquer avec les commerçants et leur } \\
\text { faire comprendre le bien-fondé de notre politique, car il } \\
\text { y a parfois des incompréhensions. » } \\
\text { "Certains pensent que seul le Groupe Lucien Barrière } \\
\text { bénéficie de cette politique. » }\end{array}$ \\
\hline Office de tourisme & $\begin{array}{l}\text { «Ce n'est pas parce qu'on s'appelle Deauville que } \\
\text { les événements et les touristes viennent à nous } \\
\text { naturellement ; nous prospectons les marchés } \\
\text { de proximité, mais aussi les marchés émergents. } \\
\text { Une douzaine de pays étrangers sont par exemple } \\
\text { prospectés en 2012. » } \\
\text { « Nos services travaillent en concertation avec de } \\
\text { nombreux acteurs, comme les institutionnels du } \\
\text { tourisme ou les acteurs privés ; ce n'est pas toujours } \\
\text { simple à faire concrètement, mais c'est nécessaire. » }\end{array}$ \\
\hline $\begin{array}{l}\text { Centre international } \\
\text { de Deauville (CID) }\end{array}$ & $\begin{array}{l}\text { «Les événements n'arrivent pas seuls. Une équipe de } \\
\text { cinq commerciaux prospecte toute l'année. » } \\
\text { « Nous sommes dans un secteur très concurrentiel } \\
\text { : d'autres villes françaises, mais aussi l'Espagne, le } \\
\text { Maghreb, l'Europe de l'Est. » }\end{array}$ \\
\hline $\begin{array}{l}\text { Pôle international } \\
\text { du cheval (PIC) et } \\
\text { hippodromes }\end{array}$ & $\begin{array}{l}\text { "Nous participons à la politique événementielle } \\
\text { de Deauville depuis peu de temps : la difficulté } \\
\text { est d'attirer des organisateurs de spectacles et de } \\
\text { compétitions et d'être capables de se renouveler sur } \\
\text { la durée.» } \\
\text { "Un renouvellement permanent des animations est } \\
\text { obligatoire et il faut donc sans cesse avoir de nouvelles } \\
\text { idées. » }\end{array}$ \\
\hline $\begin{array}{l}\text { Communauté de } \\
\text { communes }\end{array}$ & $\begin{array}{l}\text { "Deauville est souvent présentée comme superficielle } \\
\text { et c'est contre-productif. » } \\
\text { "Le système repose en grande partie sur l'énergie } \\
\text { déployée par le maire. » } \\
\text { "Depuis plusieurs dizaines d'années, il y a une } \\
\text { continuité dans les choix politiques qui sont faits ; sans } \\
\text { cela, on n'aurait pas de telles retombées, sur le plan } \\
\text { économique comme au niveau de la notoriété et de } \\
\text { l'image. » }\end{array}$ \\
\hline
\end{tabular}

(voir tableau 4). Cependant, même si les personnes interrogées s'accordent pour reconnaître que le rôle de chaque structure semble être bien défini, certains points de divergence apparaissent quand on pose la question des limites et des difficultés de la politique (voir tableau 5). Entente, coopération, transmission des informations, exigence ou effort sont des mots récurrents dans les discours. Toutefois, les responsables interrogés soulignent la condition sine qua non pour la réussite de la politique choisie : une complémentarité et une interdépendance entre les acteurs. 
ILLUSTRATION 2 : La destination touristique envisagée comme un «diamant» (source : conception des auteurs).

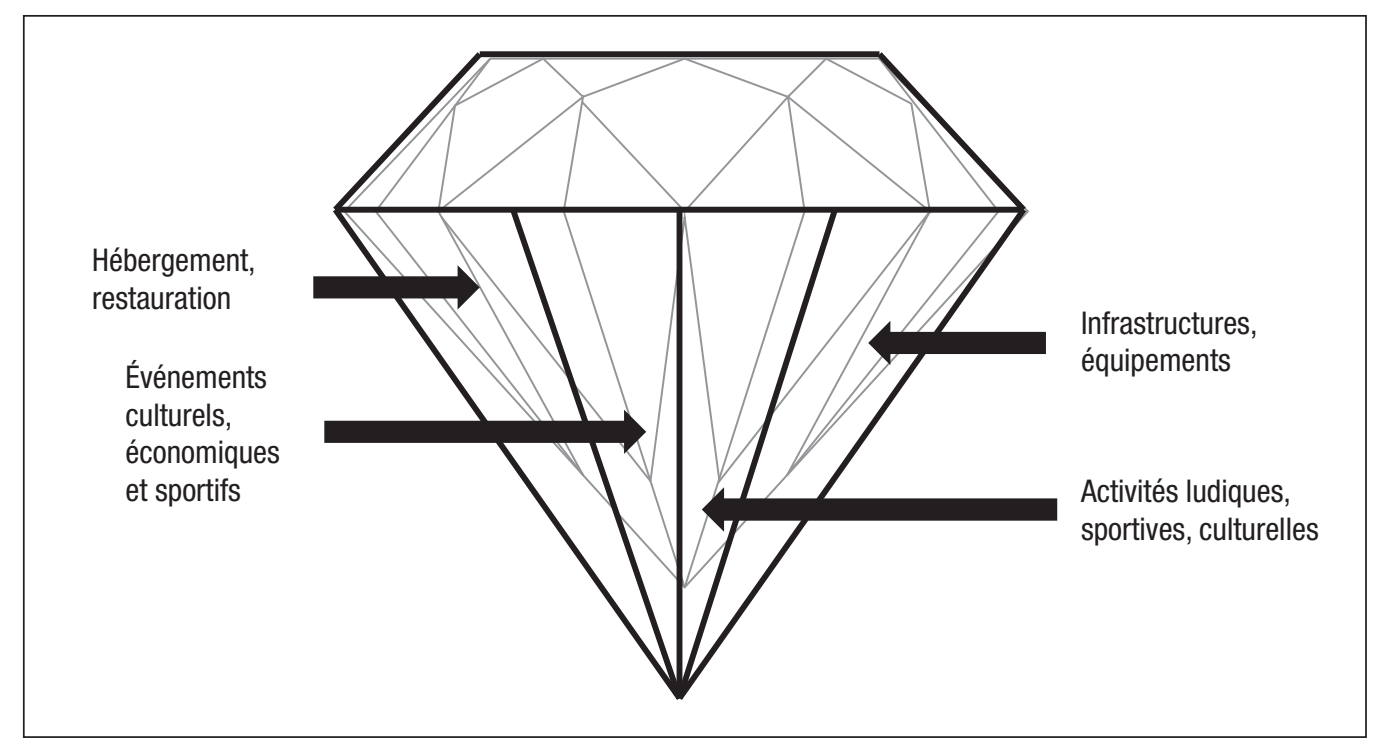

Si les extraits d'entretien ci-dessus laissent entrevoir certaines divergences d'intérêt, d'autres sont peu déclarées et plus fréquemment citées dans la presse locale. Des externalités négatives, inhérentes à l'organisation d'un événement, peuvent occasionner des problèmes. Par exemple, certains acteurs (résidents, commerçants) ne comprennent pas toujours l'enjeu d'une politique événementielle et ne relèvent que ses inconvénients : à l'occasion du G8, en 2011, alors que les responsables politiques locaux se félicitaient d'accueillir les principaux dirigeants de la planète, l'opposition municipale se faisait l'écho de certains commerçants et riverains qui dénonçaient la gêne occasionnée par l'événement. Par ailleurs, il arrive que le Groupe Lucien Barrière (propriétaire des hôtels de luxe et du casino) soit présenté comme le seul vrai bénéficiaire de cette politique. Enfin, la nécessité d'avoir toujours de nouveaux projets d'événements et d'investissement rend cette politique particulièrement exigeante. Cela passe par la prospection, ainsi que par la construction et le rajeunissement des équipements (tableau 5).

En synthèse, l'effet de levier de croissance d'une politique alliant tourisme et événement semble reconnu pour le développement d'une ville, malgré des difficultés liées à l'adhésion de tous les intervenants au projet collectif. En effet, les retombées de cette politique se manifestent à tous les niveaux : économiques, sociaux et en termes d'image. Les enseignements tirés de l'étude du cas de Deauville peuvent ainsi être discutés, voire généralisés à d'autres entités.

\section{Discussion et conclusion}

La sélection d'une destination par un touriste/visiteur peut répondre à des mobiles différents permettant de distinguer deux types d'offres :

- le choix n'est pas lié à la survenance d'une manifestation dans la station touristique. Dans ce cas, le séjour peut être agrémenté par la participation à un événement non prévue initialement. Cette activité est alors un simple additif de l'offre;
- la motivation principale est la participation à un événement. L'activité touristique se spécifie alors et devient un tourisme événementiel. Dans ce cas, l'offre de services est élaborée à partir du produit central qu'est l'événement.

Quelles que soient les motivations du touriste, les services liés à un séjour sont interconnectés. Une offre de services diversifiés et de qualité s'articule donc autour, mais aussi en dehors des événements, favorisant un contexte propice à la création de nouveaux produits et services. Le territoire est valorisé dans sa globalité : «Notre objectif est aussi d'attirer les touristes vers l'arrière-pays pour découvrir le Pays d'Auge et ses villages typiques», précise l'un des responsables de la communauté de communes.

L'illustration 2 représente de façon monolithique, sous la forme d'un diamant, les ressources qui donnent une cohérence à la destination Deauville en termes d'image et notamment aux yeux des visiteurs/spectateurs :

- des hôtels et des restaurants, en grande majorité haut de gamme (avec la présence historique du groupe hôtelier Lucien Barrière);

- une offre de loisirs (activités ludiques, sportives, culturelles, etc.);

- un grand nombre d'événements culturels, économiques et sportifs;

- des équipements d'importance et des infrastructures routières, ferroviaires et aériennes.

L'un des acteurs interrogés explique que si la taille des équipements est surdimensionnée pour une ville de moins de 4000 habitants, c'est pour répondre à sa vocation internationale : «Nous devons pouvoir accueillir des événements d'envergure (festivals et compétitions sportives internationales, notamment équestres) et mettre à la disposition de nos visiteurs une offre cohérente et de haute qualité sur tous les plans. Cela participe de notre image».

Pour un territoire comme Deauville, la représentation sous forme de "diamant » présente un intérêt dans la mesure où elle permet d'insister sur, d'une part, l'importance de 


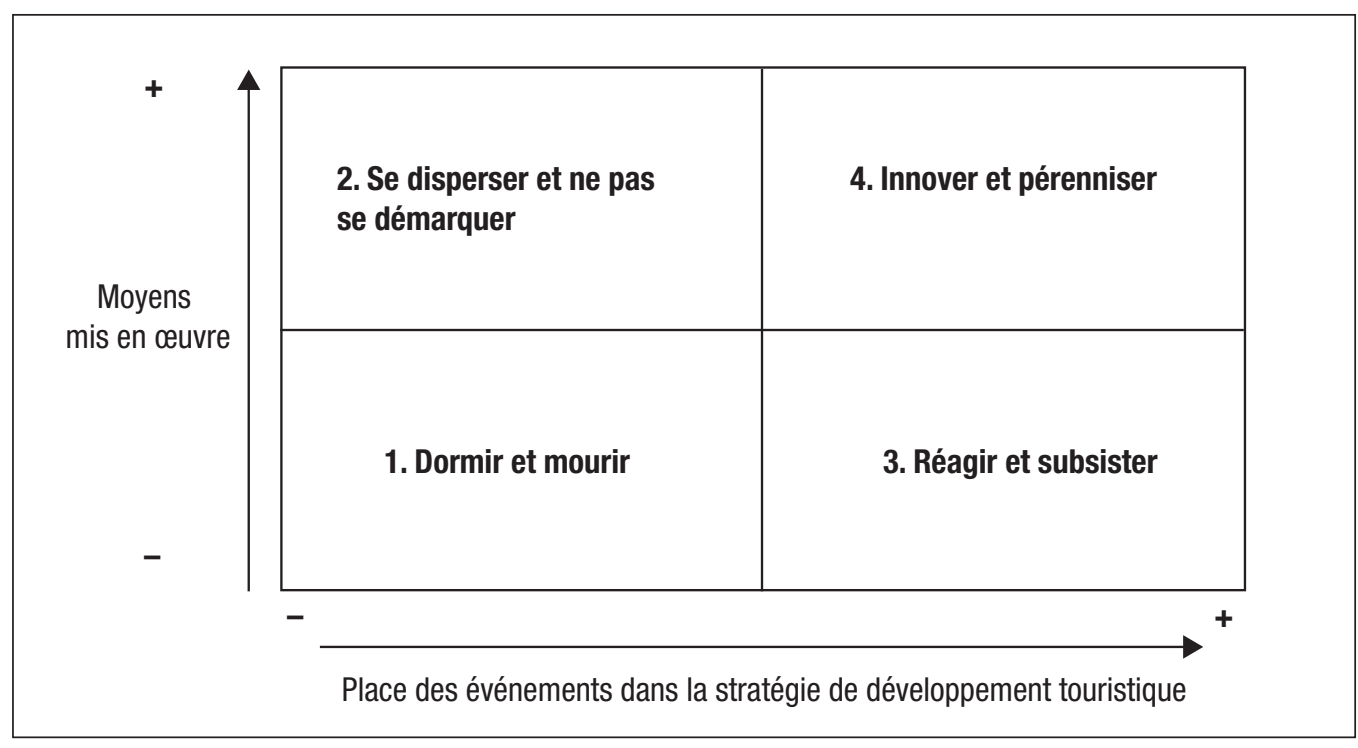

ILLUSTRATION 3 : Politiques de développement touristique par les événements (source : conception des auteurs). l'association entre les différentes facettes, porteuses de synergie et, d'autre part, le caractère unique du résultat de cette association. Tous les acteurs interrogés s'accordent pour affirmer que l'alchimie tient notamment à une spécificité du territoire : l'unité de lieu. La quasi-totalité des activités peut se dérouler au même endroit sur un espace de quelques kilomètres carrés. Ce sont les principes de facilité et de contrainte minimale qui prévalent pour une satisfaction maximale des visiteurs. Ce particularisme apporte une convivialité exceptionnelle et contribue à renforcer l'image haut de gamme de la station balnéaire et à forger ce qu'on pourrait appeler sa «légende personnelle».

L'événement peut donc être utilisé comme un levier pour amorcer «le cercle vertueux» du développement d'un territoire. En réponse aux aspirations des individus, en quête de découverte et d'émotions partagées, sont organisées des manifestations tout au long de l'année. Par exemple, à l'occasion de ses 150 ans, Deauville a été le théâtre de 365 événements culturels, au rythme d'un par jour. Les acteurs de cet anniversaire ont été les Deauvillais eux-mêmes, qu'ils soient résidents permanents ou occasionnels, invités à " coproduire» les différents rendez-vous. En encourageant cet esprit participatif, la ville a souhaité créer une dynamique : $12 \%$ des événements ont finalement été initiés par des habitants, $22 \%$ par les associations, $22 \%$ par des partenaires privés comme le Groupe Lucien Barrière ou France Galop, $16 \%$ par des entreprises et des commerçants et $28 \%$ par la Ville et ses agents (Ville de Deauville, 2010). L'année 2010, particulièrement foisonnante, ne constitue pas une exception. L'objectif affiché par les élus est que les participants de ces manifestations aient davantage envie de rester sur le territoire et prennent conscience, comme l'affirme un maire adjoint, "qu'il se passe toujours quelque chose à Deauville». Cela contribue à intensifier et désaisonnaliser la fréquentation touristique, traditionnellement concentrée sur les week-ends et les périodes de vacances scolaires.

Les politiques fondées sur l'événementiel, telles que celles proposées par la Ville de Deauville, apparaissent comme une voie originale de développement pour dynamiser un territoire, tout en lui permettant de se démarquer de ses concurrents. Le premier enseignement qu'il est possible de tirer de cette recherche, sans être original, est fondamental : il est impératif d'établir une stratégie de développement sur le long terme (Morice et Violier, 2009). L'illustration 3 présente les politiques de développement touristique possibles par les événements, mises en perspective selon deux axes : l'intensité des moyens financiers et humains mis en œuvre, d'une part, et la place des événements dans la stratégie de développement touristique, d'autre part.

Quatre cas de figure peuvent être identifiés :

- «dormir et mourir» : le territoire fait preuve d'inertie et ne vit que sur ses acquis. Telle la Belle au bois dormant, sa passivité ne débouchera que sur un déclin de son attrait, tant pour le grand public que pour les organisateurs de manifestations sportives ou culturelles;

- «se disperser et ne pas se démarquer» : la collectivité met en place ou épaule l'organisation d'événements, mais n'accorde pas une place privilégiée à cet aspect de sa politique. Un tel choix fait courir le risque de dispersion et ne lui permet pas de se démarquer de la concurrence, c'est-à-dire de ce que font toutes les autres communes;

- «réagir et subsister» : la création d'événements est alors une réponse aux attentes des touristes, qui veulent participer à des expériences collectives et vivre des émotions partagées. Si cette stratégie est réactive, elle ne conduit pas nécessairement à l'expansion par son absence de proactions et d'anticipation. Cette configuration correspond, par exemple, aux cas dans lesquels les collectivités territoriales abordent les événements comme des contraintes qu'elles sont obligées de financer;

- «innover et pérenniser» : c'est la stratégie de l'ambition, qui correspond à un système touristique de haut niveau. Il s'agit d'innover en s'appuyant sur les ressources disponibles de la commune, tout en s'adaptant au mieux à l'environnement fluctuant. 
Depuis le début des années 1990, comme le soulignent les personnes interrogées, le choix fait à Deauville a consisté à attribuer un rôle moteur aux événements. Mais plutôt que de « réagir et subsister» en organisant de nombreux événements, sans véritable fil conducteur et afin de répondre aux nouvelles aspirations des touristes, il s'agit «d'innover et pérenniser» en mettant en œuvre des moyens à la hauteur des ambitions affichées. Cette exigence implique des contraintes. Tout d'abord, il faut donner un axe fort à la politique pour garantir une cohérence sur le long terme. À Deauville, il s'agit du cheval et de la culture. Le risque de se voir accusé de «boulimie événementielle tous azimuts", et des conséquences que celle-ci entraîne (lassitude des visiteurs et des habitants à cause d'événements trop nombreux, dilution de l'image de la station), peut ainsi être évité. Parallèlement, il est nécessaire de mettre en ouvre des moyens en investissant notamment dans des infrastructures innovantes.

Il n'en demeure pas moins que la condition essentielle de la réussite d'une politique touristique basée sur les événements reste la mobilisation des énergies. Que ce soit pour le choix d'une politique touristique ou le rajeunissement d'une destination, une volonté collective forte et une réelle convergence de vues doivent s'imposer à tous les acteurs participant aux projets. Des «chefs de file» qui privilégient l'action et font preuve d'enthousiasme et d'optimisme sont absolument nécessaires pour mobiliser les forces vives existant sur un territoire (Morice et Violier, 2009; Augier, 2011). Face aux résistances susceptibles de se manifester, les élus des collectivités territoriales doivent notamment faire preuve de ténacité pour impulser la dynamique souhaitée. À l'instar de la mise en œuvre d'un projet en entreprise, celle d'une politique événementielle implique le concours d'un "promoteur», qui anime, coordonne et décide. Toutefois, selon l'envergure des événements, cette responsabilité peut incomber à des groupes d'individus regroupés en comités d'organisation. Cette structure décisionnelle impose une parfaite collaboration et communication entre ses membres, appartenant souvent à une même structure (Claveau, 2005). Dans le cas où les événements engagent plusieurs entités différentes (collectivités, entreprises, associations, etc.), c'est un comité de pilotage qui devient alors «porteur de projet».

Le mode de fonctionnement imposé à tous les acteurs, dont le centre d'intérêt commun est le même territoire, est la rencontre et la concertation. Il convient de créer des situations réunissant toutes les parties prenantes et de veiller à valoriser chacune d'elles. Au fil du temps, le projet s'étoffe et devient consensuel. Il est alors possible d'appréhender ce contexte comme un jeu stratégique, générateur d'utilité pour chaque partie. Cependant, la place des collectivités territoriales est majeure. Leur pouvoir est susceptible de s'exercer à tous les niveaux du développement touristique. La clé de la réussite d'une telle gouvernance, résultat d'une éthique de responsabilité, repose sur une volonté collective animée par un état d'esprit entrepreneurial. Ce sont des individus qui sont les moteurs de l'esprit d'entreprise collectif. Chaque acteur doit composer avec un jeu différent de contraintes et d'exigences (Akrich et al., 2006). Pour déboucher sur des résultats constructifs, les différentes parties prenantes, quelles qu'elles soient, doivent " s'apprivoiser» mutuellement, afin d'élaborer ensemble leur espace de jeu et les règles qui le structurent. Si la coopération et l'interdépendance des parties prenantes conditionnent toute proposition collective, la logique acceptée est dite déductive, c'est-à-dire de finalité et de cohérence (Crozier et Friedberg, 1981; Charrier et Rouvrais-Charron, 2009).

Une observation doit être soulignée dans le cadre de notre discussion des résultats obtenus : le type de politique de développement touristique, mis en lumière dans ce travail de recherche, n'est pas à la portée de toutes les collectivités. En effet, réflexion et action doivent se marier harmonieusement dans la durée. Or, certains territoires connaissent des faiblesses : une implication insuffisante de certains décideurs, une situation politique instable, un manque d'esprit d'entreprise des acteurs susceptibles de mettre en place des manifestations et une carence des investissements (construction et modernisation des équipements). Un accompagnement spécifique des acteurs peut, à cet égard, permettre de surmonter les obstacles. En 2010, Atout France a créé, sous l'impulsion de Philippe Augier, le maire de Deauville, une structure nationale, France Événements, dont la vocation est de faciliter l'organisation de grands événements internationaux et d'encourager les initiatives dans le cadre d'une politique globale cohérente au niveau national. Elle vise également à permettre de mieux évaluer les retombées directes et indirectes d'un événement qui restent souvent mal appréhendées a posteriori (Spindler, 2009; Gundolf et al., 2006; Maurence, 2010).

Cette contribution apporte un exemple concret de politique de développement axée sur le tourisme et l'événementiel. Notre problématique étant traitée à travers le prisme des représentations des acteurs de la ville de Deauville, notre réflexion a une portée limitée. En dépit de cette réserve, force est de constater que l'étude de tels cas concrets apporte un éclairage intéressant sur les orientations susceptibles d'être choisies pour rajeunir ou prolonger le cycle de vie d'une destination touristique.

\section{Références}

ALEXANDRE-BOURHIS Nathalie; Chantal ROUVRAIS-CHARRON et Françoise PERDRIEU-MAUDIÈRE (2009) «La valorisation des cours d'eau en Normandie : un exemple de gouvernance locale», VertigO, hors-série 6, <http://vertigo.revues.org/index8969.html>, consulté le 15 novembre 2012.

AUGIER, Philippe (2008) «La stratégie touristique de Deauville. Une politique événementielle ambitieuse et "intelligente" ", Espaces, n² 262, septembre 2008, p. 8-10.

AUGIER, Philippe (2009) Pour une politique gagnante des grands événements, Rapport au Président de la République, Présidence de la république, $79 \mathrm{p}$.

AUGIER, Philippe (2011) «Pour une politique gagnante des grands événements", Revue française du marketing, septembre 2011, n² 232, p. 61-76.

AKRICH, Madeleine; Michel CALLON et Bruno LATOUR (2006) Sociologie de la traduction. Textes fondateurs, Paris : Presses des Mines de Paris, $67 \mathrm{p}$.

BUTLER, Richard W. (1980) «The Concept of Tourism Area Cycle of Evolution: Implications for Management of Resources», Canadian Geographer, vol. 24, n 1, p. 5-12. 
Calvados Tourisme (2007) Étude de notoriété 2007, <http://www.calvadostourisme.com/fr/pros/documents/OBS-NOTORIETE-2007.pdf $>$, consulté le 29 octobre 2013.

CARON-MALENFANT Julie et Thierry CONRAUD (2009) Guide pratique de l'acceptabilité sociale : pistes de réflexion et d'action, Montréal : Éditions D.P.R.M, 60 p.

CARROLL, Archie B. et Ann K. BUCHHOLTZ (1989) Business and Society: Ethics and Stakeholder Management, Mason, Ohio : South-Western Cengage Learning, 745 p.

CARROLL, Archie B. (1999) «Corporate Social Responsibility: Evolution of a Definitional Construct», Business and Society, vol. 3, n 38, p. 268-329.

CHARRIER, Dominique et Chantal ROUVRAIS-CHARRON (2009) «La portée sociale et scientifique des recherches appliquées : contraintes et exigences d'une posture choisie», $5^{\mathrm{e}}$ congrès international de la Société française de sociologie du sport de langue française, Lyon.

CLAVEAU, Philippe (2005) Management de projets événementiels, Grenoble : Presses universitaires de Grenoble, 260 p.

CCCCF - Communauté de communes Cour Côte Fleurie (2010), «Phase 1 : Diagnostic, état initial de l'environnement \& enjeux", Document présenté dans le cadre du Séminaire PLU, 29 octobre 2013. Communauté de communes Cœur Côte Fleurie, <http://www.coeurcotefleurie.org/ Phase_1_Presentation_seminaire_01.pdf>, consulté le 29 octobre 2013.

CROZIER, Michel et Erhard FRIEDBERG (1981) L'acteur et le système : les contraintes de l'action collective, Paris : Éditions du Seuil, $493 \mathrm{p}$.

DURAND, Rodolphe (1998) «Théories évolutionnistes et management stratégique», DANS LAROCHE Hervé (sous la direction de), Repenser la stratégie, Paris : Vuibert, p. 135-165.

GANDIN, Alice (2009) Destination Normandie, deux siècles de tourisme $X I X^{e}-X X^{e} s$, Milan : 5 continents Éditions, $175 \mathrm{p}$.

GETZ, Donald (2008) «Event Tourism: Definition, Evolution, and Research ", Tourism Management, vol. 29, n 3, p. 403-428.

GHERTMAN, Michel et Guillaume ISNARD (1999) «Ressources, territoires, réseaux, un nouveau modèle de dynamique concurrentielle», Revue française de gestion, $\mathrm{n}^{\circ} 125$, septembre 1999, p. 44-57.

GUNDOLF, Katherine; Annabelle JAOUEN et Stéphanie LOUP (2006) "Institutions locales et TPE dans le cas du tourisme», Revue française de gestion, $\mathrm{n}^{\circ} 167$, p. 141-156.

HETZEL, Patrick (2002) Planète Conso, Paris : Éditions d'Organisation, 380 p.

HOLBROOK, Morris B. et Elizabeth C. HIRSCHMAN (1982) «The Experiential Aspects of Consumption: Consumer Fantasies, Feelings and Fun ", Journal of Consumer Research, vol. 9, n² 2, p. 132-139.

INSEE - Institut national de la statistique et des études économiques (2013) Populations légales 2010 pour les régions et les départements, <http:// www.insee.fr/fr/ppp/bases-de-donnees/recensement/populations-legales/>, consulté le 29 octobre 2013.
MARTEAUX, Séverine et Rémi MENCARELLI (2005) «Positionnement stratégique des entreprises culturelles : proposition d'enrichissement autour du concept de valeur», Management \& Avenir, $\mathrm{n}^{\circ}$ 5, p. 161-178.

MARCHESNAY, Michel (2002) "Les PME de terroir : entre "géo" et "clio" stratégies ", Actes de la XI conférence de l'AIMS.

MAURENCE, Éric (2010) «La mesure de l'impact économique d'un événement touristique», EMC/ministère de l'Économie, des Finances et de l'Industrie, DGCIS, décembre 2010.

MECHIN, Aude (2001) La capacité urbaine d'attraction et d'ancrage des établissements : une analyse par les ressources dynamiques, Thèse de doctorat es sciences de gestion, Université de Caen, France.

MERCIER, Samuel (2001) «L'apport de la théorie des parties prenantes au management stratégique : une synthèse de la littérature», Actes de la $X^{e}$ conférence de l'AIMS.

MORICE, Jean-René et Philippe VIOLIER (2009) «De l'événementiel culturel à la destination touristique : les cas de Lille et Nantes", Bulletin de l'Association de géographes français, vol. 86, n 3, p. 377-387.

PAILLÉ Pierre et Alex MUCCHIELLI (2003) L'analyse qualitative en sciences humaines et sociales, Paris : Éditions Armand Colin, 211 p.

OECD - Organisation for Economic Co-operation and Development (2009) The Impact of Culture on Tourism, Paris : French translation of pp.3-75, only available on Internet,

<www.oecd.org/fr/industrie/tourisme/42040218.pdf>, consulté le 15 novembre 2012 .

OTD - Office de tourisme de Deauville (2013) Les points clés qui ont renforcé l'attractivité de Deauville cette année, Deauville : Office de tourisme, $17 \mathrm{p}$.

PENROSE, Edith (1963) Facteurs, conditions et mécanismes de la croissance de l'entreprise, Neuilly-sur-Seine : Éditions Hommes et techniques, 224 p.

POLONY, Natacha (2010) «Êtes-vous Deauville ou Trouville?», Le Figaro, 12 août.

SPINDLER, Jacques (2009) L'évaluation de l'événementiel touristique, Paris : Éditions L'Harmattan, 542 p.

Ville de Deauville (2010), $1860>2010$... Deauville, le livre du cent cinquantenaire, 2010 Temps et plus, $272 \mathrm{p}$.

Ville de Deauville (2013) «Découvrir Deauville, Concours Swatch de la 25e heure», <http://www.deauville.fr/FR/agenda/1891/concours-photoswatch-de-la-25e-heure-a-deauville/?dateId=1857>, consulté le 29 octobre 2013.

WEIERMAIR, Klaus (2006) «Le vieillissement, une réalité qui s'impose aux destinations touristiques", Espaces, n 235, mars 2006, p. 18-20. 